\title{
The interplay of Piwi and heterochromatin proteins in transposable element silencing in the germline of Drosophila melanogaster
}

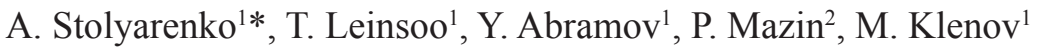 \\ ${ }^{1}$ Institute of Molecular Genetics RAS, Moscow, Russia \\ ${ }^{2}$ Center for Data-Intensive Biomedicine and Biotechnology, Skolkovo Institute of Science and Technology, \\ Moscow, Russia \\ *e-mail:stol@img.ras.ru
}

Key words: Piwi, Argonaute, HP1a, heterochromatin, transcriptional silencing, RNAseq

Motivation and Aim: Transposable element (TE) silencing is critical for germline genome stability and fertility of animals, including Drosophila melanogaster. The piRNAbinding protein Piwi of the Argonaute family was shown to induce transcriptional repression of TEs in Drosophila ovaries [1]. However, the interplay of Piwi and the main heterochromatin protein HP1a and other heterochromatin proteins in this process is still not fully understood, and Piwi is usually considered to team with HP1a to carry out TE repression [2].

Methods and Algorithms: We took advantage of a genetic approach of combining in one genotype germline knockdowns (KD) of Piwi and HP1a and comparing them with single Piwi and HP1a KDs in the same genetic background via RNA-seq. We divided TEs responding to the double KD according to their response to Piwi and HP1a KDs into four groups.

Results: A group of TEs are equally controlled by Piwi or HP1a which points to the participation of these two proteins in a single pathway to silence the elements of this group. Another group of TEs are controlled by Piwi alone, but not HP1a. Another group of elements are derepressed only upon double KD. This implies independent double control by systems based on Piwi and HP1a in combination. Interestingly, according to our previously published ChIP-seq data, the chromatin of these TEs is especially highly enriched in the repressive $\mathrm{H} 3 \mathrm{~K} 9 \mathrm{me} 3$ mark, which is indicative of their localization in heterochromatin. Finally, there is a group of Piwi-independent elements controlled by HP1a alone. Interestingly, we found that these elements coincided with the ones known to be derepressed upon mutation of endosiRNA-binding protein Ago2 [3]. We propose that the repression of these elements by Ago 2 is assisted by HP1a in the germline.

Conclusion: Our results indicate that Piwi-induced silencing and Piwi-independent repression by heterochromatic proteins may represent two distinct systems that silence transcription of TEs in the ovarian germline. These two systems can act autonomously or perhaps in association with each other to repress an element.

Acknowledgements: Supported by the RFBR (16-04-01524).

References

1. Klenov M. et al. (2007) Repeat-associated siRNAs cause chromatin silencing of retrotransposons in the Drosophila melanogaster germline. Nucleic Acids Res. 35(16):5430-5438.

2. Sienski G. et al. (2015) Silencio/CG9754 connects the Piwi-piRNA complex to the cellular heterochromatin machinery. Genes Dev. 29(21):2258-2271.

3. Czech B. et al. (2008) An endogenous small interfering RNA pathway in Drosophila. Nature. 453(7196): 798-802. 